Hautarzt 2009 -60:794-794

DOI 10.1007/s00105-009-1758-5

(C) Springer Medizin Verlag 2009

\section{J. Krutmann}

Institut für Umweltmedizinische Forschung (IUF) an der

Heinrich-Heine-Universität Düsseldorf gGmbH

\title{
Pro- und Präbiotika in der Dermatologie
}

Die Forschung der letzten Jahren hat gezeigt, dass die Mikroflora (Mikrobiota), die Barrierefunktion und das angeborene Immunsystem der Haut sich gegenseitig in einem komplexen Wechselspiel beeinflussen können. Hieraus ergibt sich folgerichtig ein großes Interesse an der Entwicklung neuer Strategien, mit denen es möglich ist, die Mikroflora der Haut in ihrer Komposition zu beeinflussen und zwar selektiver, als dies durch antibiotische Strategien möglich ist. Neben diesen präbiotischen Ansätzen zeichnet sich zudem ab, dass sowohl topische als auch insbesondere systemische probiotische Strategien für die Hautgesundheit relevant sind.

\section{(7) Sowohl topische als auch systemische probiotische Strategien sind für die Hautgesundheit relevant}

In dem hier vorliegenden Leitthemenheft wird ein umfassender Überblick über diese Entwicklungen gegeben. Sie reichen von der Entwicklung prä- und probiotischer kosmetischer Ansätze über den Einsatz systemischer Probiotika zur Behandlung entzündlicher Hautkrankheiten wie der atopischen Dermatitis bis hin zur Entwicklung probiotischer Externa. Das Wissen um diese Ansätze ist gerade auch für den Praktiker von Bedeutung, da Patienten in zunehmendem Maße in allen Lebensbereichen mit diesen Entwicklungen konfrontiert werden, so z. B. beim Lebensmitteleinkauf mit probiotischen Nahrungsmittelprodukten, die als funktionelle Nahrungsmittel für die Haut angeboten werden. Die Forschung und Entwicklung aber auch die klinische Anwendung stehen hier erst am Anfang, allerdings weisen die ersten Ergebnisse daraufhin, dass es sich hierbei um zum Teil außerordentlich wirkungsvolle und bezüglich ihrer Wirkmechanismen hoch interessante Verfahren handelt.

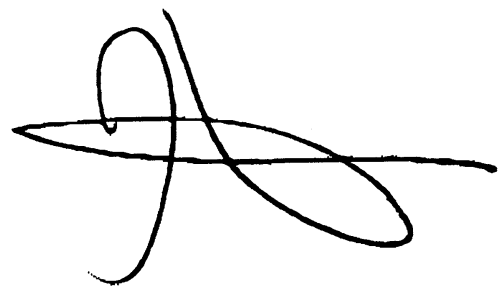

Prof. Dr. Jean Krutmann

\section{Korrespondenzadresse \\ Prof. Dr. med. Jean Krutmann

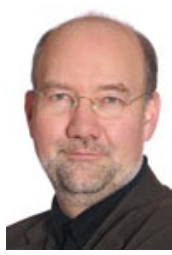 Institut für Umweltmedizi- nische Forschung (IUF) an der Heinrich-Heine-Univer- sität Düsseldorf gGmbH Auf'm Hennekamp 50 40225 Düsseldorf krutmann@rz.uni- duesseldorf.de}

\section{Weiterführende Literatur}

1. Krutmann J: Pre- and Probiotics for Human Skin. J Dermatol Sci 54:1-5, 2009.

2. Krutmann J, Humbert $P$ (eds): Nutrition for Healthy Skin: Strategies for Clinical and Cosmetic Practice. Springer-Verlag, Berlin, New York, 2009 (in press) 